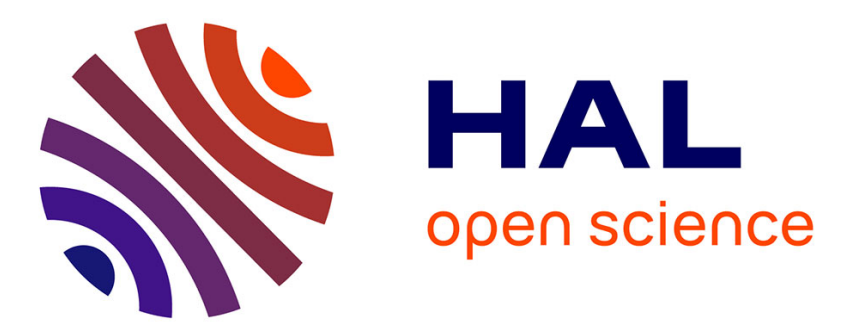

\title{
Rational Approximation of Transfer Functions for Non-Negative EPT Densities
}

Conor Sexton, Martine Olivi, Bernard Hanzon

\section{To cite this version:}

Conor Sexton, Martine Olivi, Bernard Hanzon. Rational Approximation of Transfer Functions for Non-Negative EPT Densities. 16th IFAC Symposium on System Identification, Jul 2012, Bruxelles, Belgium. pp.716-721, 10.3182/20120711-3-BE-2027.00197 . hal-00763205

\section{HAL Id: hal-00763205 https://hal.inria.fr/hal-00763205}

Submitted on 10 Dec 2012

HAL is a multi-disciplinary open access archive for the deposit and dissemination of scientific research documents, whether they are published or not. The documents may come from teaching and research institutions in France or abroad, or from public or private research centers.
L'archive ouverte pluridisciplinaire HAL, est destinée au dépôt et à la diffusion de documents scientifiques de niveau recherche, publiés ou non, émanant des établissements d'enseignement et de recherche français ou étrangers, des laboratoires publics ou privés. 


\title{
Rational Approximation of Transfer Functions for Non-Negative EPT Densities
}

\author{
Conor Sexton ${ }^{*, 1}$ Martine Olivi ${ }^{* *}$ Bernard Hanzon ${ }^{* * *, 2}$ \\ ${ }^{*}$ Edgeworth Centre for Financial Mathematics, School of Mathematical \\ Sciences, University College Cork, Western Rd., Cork, Ireland (e-mail: \\ hcsexton@hotmail.com) \\ ** INRIA, 2004 route des Lucioles, BP 93, 06902, Sophia Antipolis, Cedex, \\ France (e-mail: Martine.Olivi@sophia.inria.fr \\ ${ }^{* * *}$ Edgeworth Centre for Financial Mathematics, School of Mathematical \\ Sciences, University College Cork, Western Rd., Cork, Ireland (e-mail: \\ b.hanzon@ucc.ie)
}

\begin{abstract}
An Exponential-Polynomial-Trigonometric (EPT) function is defined on $[0, \infty)$ by a minimal realization $(\mathbf{A}, \mathbf{b}, \mathbf{c})$. A stable non-negative EPT function of a fixed degree is fitted to the histogram of a large set of data using an $L^{2}$ criterion. If we neglect the non-negativity constraint this is shown to be equivalent to a rational approximation problem which is approached using the RARL2 software. We show how, under the additional assumption of the existence of a strictly dominant real pole of the rational function, the non-negativity constraint on the EPT function can be imposed by performing a constraint convex optimization on $\mathbf{b}$ at each stage at which an $(\mathbf{A}, \mathbf{c})$ pair is determined. In this convex optimization step a recent generalized Budan-Fourier sequence approach to determine non-negativity of an EPT function on a finite interval plays a major role.
\end{abstract}

Keywords: Probability Density Function, Rational Matrices, Fourier Transforms, Impulse Responses, Lyapunov Function, Convex Optimisation, Function Approximation, Sampled Data, Financial Systems

\section{Introduction}

The flexible class of 2-EPT probability density functions on $(-\infty, \infty)$ are introduced in Sexton and Hanzon [2012] where it is illustrated how they provide a useful framework for univariate probabilistic calculations. Here we consider the subclass of EPT probability density functions. These are defined on $[0, \infty)$. These are non-negative functions of the form $\mathbf{c} e^{\mathbf{A} x} \mathbf{b}$, with $(\mathbf{A}, \mathbf{b}, \mathbf{c})$ minimal, $\mathbf{A}$ asymptotically stable and with integral equal to one. Similarly EPT functions can be used to model forward interest rates as in Hanzon and Holland [2010] where non-negativity is equally important. The problem of approximating empirical data with a non-negative EPT function is considered here. Despite the fact that the observed data may be non-negative it is still possible that the approximating EPT function is negative on certain intervals. This problem has been observed in practice and is also encountered in model reduction when approximating a high order non-negative system with a lower order system.

Anderson et al [1996] introduce positive realizations to ensure positive impulse responses. A positive realization is characterised by non-negativity of all elements of the discrete time stable realization. However positive realizations may not always exist for an externally positive system and if such a realization does exist then it may be non-minimal (i.e. it may be nonobservable and/or non-reachable).

We demonstrate how to fit a minimal non-negative EPT density

\footnotetext{
1 Supported by Science Foundation Ireland under grant 07/MI/008.

2 Supported by Science Foundation Ireland under grant numbers 07/MI/008 and RFP2007-MATF802.
}

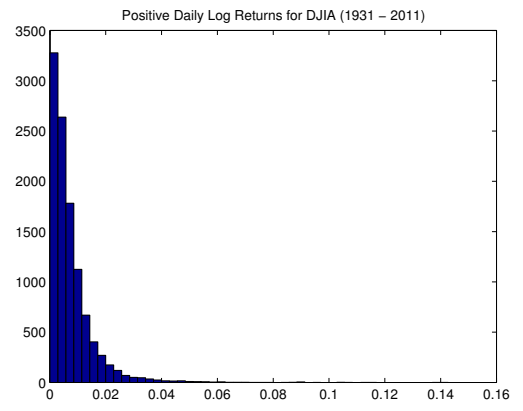

Fig. 1. Daily Log returns for DJIA for (1931 - 2011)

on a particular set of data. The dataset to be approximated is the positive daily Dow Jones Industrial Average (DJIA) log returns over 80 years which are shown in Figure 1. (Fitting another EPT function on $(-\infty, 0]$ to the negative log returns can be carried out by the same procedure). Hence the goal of the paper is to describe a procedure to find a triple $(\mathbf{A}, \mathbf{b}, \mathbf{c}) \in \mathbf{R}^{n \times n} \times \mathbf{R}^{n \times 1} \times$ $\mathbf{R}^{1 \times n}$ of McMillan degree $n$ minimizing the criterion

$$
\left\|h(x)-\mathbf{c} e^{\mathbf{A} x} \mathbf{b}\right\|_{2}^{2}
$$

where $h(x)$ is a density function that represents the data $(h(x)$ is directly related to a histogram of the data; a precise description of $h$ is given in Section 2). Here the norm is the $L^{2}$ norm over the half-line $[0, \infty)$.

The EPT function can alternatively be interpreted as the impulse response of a continuous time stable system whose Laplace transform is a rational transfer function. This interpretation will be useful as it allows us to apply methods from 
systems theory to the problem at hand.

Section 2 shows how the tail of the function can be estimated. The necessity of a dominant real pole in the spectrum of $\mathbf{A}$ is also discussed. Section 3 illustrates that our $L^{2}$ fitting problem is equivalent to a discrete time rational approximation problem in $H^{2}$. The unconstrained problem, in which the non-negativity constraint is not imposed, is tackled using the rational approximation software RARL2 to find an observable pair $(\mathbf{A}, \mathbf{c})$ and an optimal $\mathbf{b}$. In a separate section, Section 4, and in Appendix A, the Fourier coefficients of the transform of $h(x)$, which form the inputs to RARL2, are derived directly as inner products of $h(x)$ with a set of orthogonal basis functions on $[0, \infty)$. Results from unconstrained RARL2 approximations are also provided.

The non-negativity problem is described in Section 5. By assuming the presence of a dominant real pole and solving the unconstrained problem with RARL2 we show that non-negativity can be imposed by choosing an optimal $\mathbf{b}_{C}^{*}$ for a given pair $(\mathbf{A}, \mathbf{c})$ such that the EPT function with realization $\left(\mathbf{A}, \mathbf{b}_{C}^{*}, \mathbf{c}\right)$ is non-negative on $[0, \infty)$. Choosing $\mathbf{b}_{C}^{*}$ is shown to be a convex optimisation problem which is solved in Section 6. Central to the convex optimisation algorithm is the Budan-Fourier method of Hanzon and Holland [2010] which examines non-negativity of an EPT function on a finite interval $[0, T]$. Section 7 proves that a finite interval can be constructed on which to apply the Budan-Fourier technique to obtain a necessary and sufficient test for non-negativity of the EPT function on $[0, \infty)$. This is possible due to the assumed presence of a strictly dominant real pole in the spectrum of $\mathbf{A}$. Finally Section 8 gives an example of the convex optimisation procedure.

\section{Extrapolating the Tail}

We take a histogram of daily log returns for DJIA for the period 1931-2011 as our starting point (see Figure 1). Firstly we scale the data such that all data points are located in the interval $[0,1]$. We denote the resulting function by $r(x)$. This is a step function. As its support is only on the finite interval $[0,1]$ we extrapolate the tail of the empirical density to the whole positive real line with an exponential function which is assumed to contain the strictly dominant real pole of the function. One (regression) technique of identifying the strictly dominant real pole and its coefficient is to select $K$ points on the histogram $r(x)$ and choose the parameters $\mu_{M}>0$ and $\lambda_{M}<0$ to minimise

$$
\min _{\mu_{M}, \lambda_{M}} \sum_{i=N-K}^{N}\left(\log \left(r\left(x_{i}\right)\right)-\log \left(\mu_{M} e^{\lambda_{M} x_{i}}\right)\right)^{2}
$$

where $x_{0}=0, x_{N}=1$ and $x_{i+1}-x_{i}=\delta x$ for all $i$ where $\delta x=\frac{1}{N}$. $K$ must be chosen to capture tail behaviour only, so should not be chosen too big, but also not too small, in order to get a good estimate of $\mu_{M}$ and $\lambda_{M}$. In this financial setting it makes particular sense to estimate the tail behaviour of the histogram $r(x)$ separately, as data is sparse in this region of the distribution. We then define the function $h(x)$ on $[0, \infty)$ as

$$
h(x)= \begin{cases}r(x) & \text { if } \mathrm{x} \leq 1 \\ \mu_{M} e^{\lambda_{M} x} & \text { if } \mathrm{x}>1\end{cases}
$$

In Figure 2 we can see $h(x)$ on $[0,0.3]$.

As we proceed to examining non-negative EPT approximations we elucidate to the importance of the Perron-Frobenius type result which states that if $f(x)=\boldsymbol{c} e^{\boldsymbol{A} x} \boldsymbol{b}$ is non-negative $\forall x \geq 0$ then $\lambda_{M}=\max _{\lambda \in \sigma(A)} \operatorname{Re}(\lambda)$ is an element of the spectrum of $\boldsymbol{A}$. The spectrum of $\mathbf{A}$ is denoted $\sigma(\mathbf{A})$. This result puts a constraint on the eigenvalues of the approximating A matrix in that it must contain a dominant real eigenvalue (equivalently the rational characteristic function must contain a dominant real pole). This condition is far from sufficient and as the analysis in Hanzon and Holland [2011] shows the nonnegativity constraint is a complicated one in general.

We do not impose this condition on $\sigma(\mathbf{A})$ in Sections 3 or 4 , instead allowing RARL2 to recover the dominant real pole during the approximation procedure.

\section{Discrete Time Rational Approximation with RARL2}

As is well-known minimizing Eq. (1) is equivalent to minimizing the $L_{2}(i \mathbf{R})$ distance between the characteristic functions (Fourier transforms) of $h(x)$ and $f(x)$, where $f(x)=\mathbf{c} e^{\mathbf{A} x} \mathbf{b}$. The characteristic function of $\mathbf{c}^{\mathbf{A} x} \mathbf{b}$ is a rational function with poles in the open left half plane and fixed McMillan degree. In this way the problem of minimizing Eq. (1) can be translated into a rational approximation problem under a "least squares" norm. In systems theory this problem is known as the $H_{2}$ model approximation problem for continuous time systems. The software RARL2 is devised to handle similiar problems for discrete time models. We use an isometric transformation to map the continuous time system into a discrete time system and then apply the software.

RARL2 is ideally suited to this rational approximation problem as it uses a parameterization of the observable pairs $(\mathbf{A}, \mathbf{c})$ which is convenient for enforcing the stability constraint and for optimisation due to their differentiability properties. The inputs to RARL2 are a finite number of Fourier coefficients of the transfer function of the discrete-time system. RARL2 was developed at INRIA and a description with further references can be found in Olivi [2010]. Without the non-negativity constraint the criterion function to be minimised is Eq. (1) over the set of minimal triples $(\mathbf{A}, \mathbf{b}, \mathbf{c})$ where $\mathbf{A}$ is stable and of fixed degree $n$.

The continuous time transfer function is found via the transform

$$
\mathbb{E}\left[e^{-s x}\right]=F(s)=\int_{0}^{\infty} \mathbf{c} e^{\mathbf{A} x} \mathbf{b} e^{-s x} d x=\mathbf{c}(s \mathbf{I}-\mathbf{A})^{-1} \mathbf{b}
$$

which is a strictly proper rational function. The transform of $h(x)$ is given by

$$
H(s)=\frac{\mu_{M} e^{\lambda_{M}-s}}{s-\lambda_{M}}+\sum_{n=0}^{N-1} r\left(x_{n}\right)\left(-\frac{1}{s} e^{-s x_{n+1}}+\frac{1}{s} e^{-s x_{n}}\right)
$$

To transform the continuous time rational function into discrete time we use a well known isometry. In discrete time the stable A matrix will have all its eigenvalues located inside the unit disk. The map from continuous to discrete time is

$$
F(s) \mapsto \tilde{F}(z)=\frac{\sqrt{2}}{z-1} F\left(\frac{z+1}{z-1}\right)
$$

achieved using the Möbius transform

$$
z \mapsto s=\frac{z+1}{z-1}
$$

These formulae allow us to derive a discrete time realization, $(\tilde{\mathbf{A}}, \tilde{\mathbf{b}}, \tilde{\mathbf{c}})$, from the continuous time realization $(\mathbf{A}, \mathbf{b}, \mathbf{c})$. The state space formulae of the transformation are given by

$$
\begin{aligned}
\tilde{\mathbf{A}} & =-(\mathbf{I}-\mathbf{A})^{-1}(\mathbf{I}+\mathbf{A}) \\
\tilde{\mathbf{b}} & =\sqrt{2}(\mathbf{I}-\mathbf{A})^{-1} \mathbf{b} \\
\tilde{\mathbf{c}} & =\mathbf{c}
\end{aligned}
$$


Hence the discrete time rational function is given by

$$
\tilde{F}(z)=\tilde{\mathbf{c}}(z \mathbf{I}-\tilde{\mathbf{A}})^{-1} \tilde{\mathbf{b}}
$$

Equations (6) and (7) can be used to transform $H(s)$ into its discrete time counterpart $\tilde{H}(z)$. We let

$$
i \omega=\frac{e^{i \theta}+1}{e^{i \theta}-1}, \theta \in[-\pi, 0) \cup(0, \pi]
$$

giving us

$$
\begin{aligned}
\tilde{H}\left(e^{i \theta}\right) & =\frac{\sqrt{2}}{e^{i \theta}-1} H(i \omega)=\left(\frac{\sqrt{2}}{e^{i \theta}-1}\right) \frac{\mu_{M} e^{\lambda_{M}-i \omega}}{i \omega-\lambda_{M}} \\
& +\frac{\sqrt{2}}{e^{i \theta}-1}\left(\sum_{n=0}^{N-1} \hat{r}\left(x_{n}\right)\left(\frac{1}{i \omega} e^{-i \omega x_{n}}-\frac{1}{i \omega} e^{-i \omega x_{n+1}}\right)\right)(11)
\end{aligned}
$$

RARL2 seeks to minimise the $L_{2}$ norm

$$
\min _{\tilde{\mathbf{A}}, \tilde{\mathbf{b}}, \tilde{\mathbf{c}}}\|\tilde{H}-\tilde{F}\|_{2}^{2}
$$

which is equivalent to Eq. (1).

\section{RARL2 Approximation from Fourier Coefficients}

Let $\left\{g_{n}(x)\right\}_{n=-1,-2,-3, \ldots}$ denote the sequence of functions for $n<0$ given by

$$
g_{n}(x)=\sqrt{2} e^{-x} \sum_{j=0}^{|n|-1}\left(\begin{array}{c}
-n-1 \\
j
\end{array}\right) \frac{(-2 x)^{j}}{j !}, x \in[0, \infty)
$$

which form an orthonormal basis for the Hilbert space $L^{2}[0, \infty)$ of real square integrable functions on $[0, \infty)$. The function $h(x)$ can then be represented by a weighted series of orthonormal functions

$$
h(x)=\sum_{n<0} c_{n} g_{n}(x)
$$

The coefficients are given by

$$
c_{n}=<g_{n}, h>=\int_{0}^{\infty} g_{n}(x) h(x) d x
$$

which can be obtained by computing the integral in Eq. (15), numerically on $[0,1]$ and analytically on $[1, \infty)$. It is proven in Appendix $\mathrm{A}$ that the coefficients $c_{n}$ are the Fourier coefficients of the discrete time function $\tilde{H}$. It is clear that $g_{n}$ is an Exponential-Polynomial (EP) function and subsequently the approximation of $h(x)$ derived from a truncated $\operatorname{sum} \sum_{n=-1,-2, \ldots,-M} c_{n} g_{n}(x)$ is also EP.

The RARL2 software then uses these Fourier coefficients to determine a high order rational approximant and to calculate a first approximation of this in the form of the discrete time minimal system of degree $n$. This is then used as a starting point for the RARL2 approximation which uses a gradient search algorithm to locate a local minimum, in the form of a minimal realization of degree $n$, of the criterion in Eq. (12). The algorithm will report the smallest local minimum found.

By calculating the Fourier coefficients of $h(x)$ as described in Eqs. (13) to (15) we evaluate the performance of two RARL2 approximations, one of order 3 and another of order 6 . Figure 2 illustrates the normalised empirical data, $h(x)$, (integrates to unity) and the RARL2 approximations in continuous time.

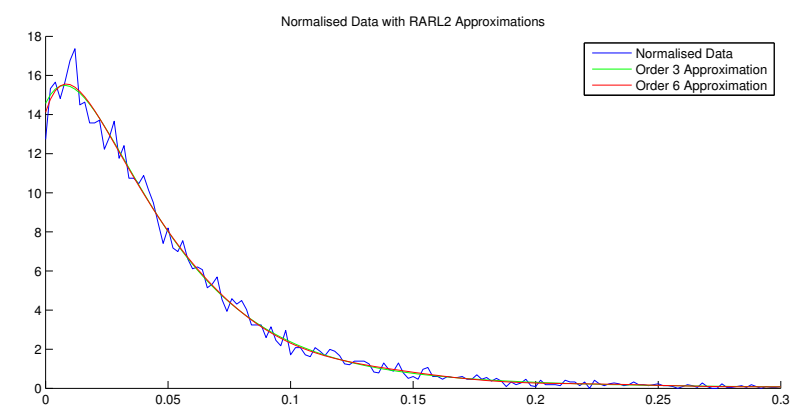

Fig. 2. The Density Function $h(x)$ is in blue. RARL2 Approximations of Order 3 (green) and of Order 6 (red)

The approximation of order 3 has a square error of 0.0501 compared with the order 6 approximation which has a square error of 0.0441 , demonstrating the improvement of fit using higher order approximations. The poles of the rational transfer function of the order 3 approximation are $(-11.866,-38.474 \pm$ $14.297)$ while the zeros are $(-15.330,-88.617)$. The order 3 approximation contains a dominant real pole of -11.866 . Using techniques described in Section 7 we conclude that this approximation is non-negative for all $x>0$. The poles of the order 6 rational transfer function are $(-9.388 \pm$ $71.265,-12.478 \pm 4.594,-24.972,-76.060)$ and the zeros are $(-130.329,-9.054 \pm 71.465,-12.209 \pm 5.465)$. However the dominant pole of the order 6 approximation is complex and the resulting EPT is negative on certain intervals for $x>0.71$.

From Section 2 the dominant real pole was estimated as $\lambda_{M}=$ -10.956 with its coefficient $\mu_{M}=3.023$.

\section{Toward a Non-Negative EPT Approximation}

If applying unconstrained the method as described in Sections 3 and 4 using RARL2 produces an EPT function with negative values on $x \geq 0$ then we must alter our approach to ensure nonnegativity. An important tool is the generalized Budan-Fourier algorithm of Hanzon and Holland [2010] can be used to test for non-negativity of an EPT function on a finite interval $[0, T]$. For each real EPT function on a finite interval $[0, T] \subset \mathbb{R}$ a generalized Budan-Fourier sequence with associated sets of boundary points can be constructed. The construction is given directly in terms of the parameters of the EPT function. The generalized Budan-Fourier sequence with associated sets of boundary points, allows one to find the minimum of the EPT function on $[0, \mathrm{~T}]$ by repeatedly using bisection techniques. This allows one to characterize non-negative EPT functions.

Again $h(x)$ is the true function we are approximating with the EPT function $f(x)=\mathbf{c} e^{\mathbf{A} x} \mathbf{b}$ of McMillan degree $n$, the minimization problem with the non-negativity constraint we wish to solve is given by Eq. (1).

Recalling the Perron-Frobenius result from Section 2 we will now assume the presence of a unique strictly dominant real eigenvalue, $\lambda_{M}<0$, of multiplicity one in the spectrum of $\mathbf{A}$ (so all other eigenvalues in the spectrum have real part strictly less than $\lambda_{M}$ ). This assumption may be slightly restrictive as the Perron-Frobenius result allows for multiple dominant eigenvalues. The strictly dominant eigenvalue and its coefficient, $\mu_{M}$, are estimated as shown in Section 2.

The dominant eigenvalue and coefficient can then be handled separately from the other parameters in the approximation by transforming $h(x)$ and $f(x)$ as follows 


$$
\begin{aligned}
& \hat{h}(x)=h(x)-\mu_{M} e^{\lambda_{M} x} \\
& \hat{f}(x)=f(x)-\mu_{M} e^{\lambda_{M} x}=\hat{\mathbf{c}} e^{\hat{\mathbf{A}} x} \hat{\mathbf{b}}
\end{aligned}
$$

where $\hat{f}(x)=\hat{\mathbf{c}} e^{\hat{\mathbf{A}} x} \hat{\mathbf{b}}$ is an EPT function of McMillan degree $(n-1)$.

To ensure $\lambda_{M}$ remains the dominant pole of $f(x)$ the eigenvalues of $\hat{\mathbf{A}}$ are constrained in the approximation such that $\operatorname{Re}(\sigma(\hat{\mathbf{A}}))<\lambda_{M}$. This restriction on $\sigma(\hat{\mathbf{A}})$ is similar to the stability condition and can be enforced in RARL2.

As noted already, the RARL2 algorithm so far does not take the non-negativity constraint into consideration. Implementing RARL2 after the above transformations yields the criterion

$$
\left\|\hat{h}(x)-\hat{\mathbf{c}} e^{\hat{\mathbf{A}} x} \hat{\mathbf{b}}\right\|_{2}^{2}
$$

for which a local minimum is found using the gradient algorithms described in Marmorat and Olivi [2007] where the pair $(\hat{\mathbf{A}}, \hat{\mathbf{c}})$ range over the set of output normal observable pairs as described in Hanzon et al [2006]. For a given pair $(\hat{\mathbf{A}}, \hat{\mathbf{c}})$ it is a linear problem to solve for the optimal $\hat{\mathbf{b}}^{*}$. The Fourier coefficients of $\hat{h}(x)$ may be computed by evaluating the integral in Eq. (15) on $[0,1]$ as $\hat{h}(x)=0$ for all $x>0$.

The non-empty convex set $\mathcal{B}(\hat{\mathbf{A}}, \hat{\mathbf{c}})$ can then be defined for any pair $(\hat{\mathbf{A}}, \hat{\mathbf{c}})$ such that if $\hat{\mathbf{b}}^{*} \in \mathcal{B}(\hat{\mathbf{A}}, \hat{\mathbf{c}})$ then non-negativity is guaranteed by $f(x) \geq 0$ or equivalently

$$
\hat{f}(x)=\hat{\mathbf{c}} e^{\hat{\mathbf{A}} x} \hat{\mathbf{b}}^{*} \geq-\mu_{M} e^{\lambda_{M} x} \quad \forall x \geq 0
$$

However if $\hat{\mathbf{b}}^{*} \notin \mathcal{B}(\hat{\mathbf{A}}, \hat{\mathbf{c}})$ then using the convex optimisation algorithm, described in Section 6 , it is possible to find the constrained optimal $\hat{\mathbf{b}}_{C}^{*} \in \mathcal{B}(\hat{\mathbf{A}}, \hat{\mathbf{c}})$ s.t. $\hat{\mathbf{b}}_{C}^{*}$ is the solution for $\hat{\mathbf{b}}$ to the minimization problem

$$
\min _{\{\hat{\mathbf{b}} \in \mathcal{B}(\hat{\mathbf{A}}, \hat{\mathbf{c}})\}}\left\|\hat{h}(x)-\hat{\mathbf{c}} e^{\hat{\mathbf{A}} x} \hat{\mathbf{b}}\right\|_{2}^{2}
$$

The final triple for the non-negative EPT function $f$ can represented in state space form by

$$
\left(\begin{array}{cc|c}
\lambda_{M} & 0 & 1 \\
0 & \hat{\mathbf{A}} & \hat{\mathbf{b}}_{C}^{*} \\
\hline \mu_{M} & \hat{\mathbf{c}} & 0
\end{array}\right)
$$

\section{Convex Optimisation with the generalized Budan Fourier Algorithm}

Consider the EPT function

$$
f(x)=\mu_{M} e^{\lambda_{M} x}+\hat{\mathbf{c}} e^{\hat{\mathbf{A}} x} \hat{\mathbf{b}}
$$

as described in Section 4. If $\hat{\mathbf{b}}=\hat{\mathbf{b}}^{*} \notin \mathcal{B}(\hat{\mathbf{A}}, \hat{\mathbf{c}})$ then the function $f(x)$ will have negative values for some $x \geq 0$.

We intend to apply a convex optimisation algorithm to solve the minimization problem

$$
\min _{\{\hat{\mathbf{b}} \in \mathcal{B}(\hat{\mathbf{A}}, \hat{\mathbf{c}})\}}\left\|\hat{\mathbf{c}} e^{\hat{\mathbf{A}} x}\left(\hat{\mathbf{b}}^{*}-\hat{\mathbf{b}}\right)\right\|_{2}^{2}
$$

We see that Eq. (19) can be re-written as

$$
\begin{aligned}
& \left\|\hat{h}(x)-\hat{\mathbf{c}} e^{\hat{\mathbf{A}} x} \hat{\mathbf{b}}^{*}+\hat{\mathbf{c}} e^{\hat{\mathbf{A}} x} \hat{\mathbf{b}}^{*}-\hat{\mathbf{c}} e^{\hat{\mathbf{A}} x} \hat{\mathbf{b}}\right\|_{2}^{2} \\
= & \left\|\hat{h}(x)-\hat{\mathbf{c}} e^{\hat{\mathbf{A}} x} \hat{\mathbf{b}}^{*}\right\|_{2}^{2}+\left\|\hat{\mathbf{c}} e^{\hat{\mathbf{A}} x}\left(\hat{\mathbf{b}}^{*}-\hat{\mathbf{b}}\right)\right\|_{2}^{2}
\end{aligned}
$$

which is possible due to the orthogonality of $\left(\hat{h}(x)-\hat{\mathbf{c}} e^{\hat{\mathbf{A}} x} \hat{\mathbf{b}}^{*}\right)$ and $\hat{\mathbf{c}} e^{\hat{\mathbf{A}} x}\left(\hat{\mathbf{b}}^{*}-\hat{\mathbf{b}}\right)$ as the triple $\left(\hat{\mathbf{A}}, \hat{\mathbf{b}}^{*}, \hat{\mathbf{c}}\right)$ is the projection of $\hat{h}$ onto the vector space $\left\{\hat{\mathbf{A}}, \beta, \hat{\mathbf{c}} \mid \beta \in \mathbb{R}^{n-1}\right\}$. Hence it is clear that minimising the criterion in Eq. (19) is equivalent to minimising the criterion in Eq. (21).

Section 7 derives a $T_{0}$ such that if the EPT function is nonnegative on $\left[0, T_{0}\right]$ then non-negativity is assured on $[0, \infty)$. This is possible due to the presence of the real strictly dominant pole and a restriction on the norm of $\hat{\mathbf{b}}$. Then the generalized Budan-Fourier algorithm can determine whether non-negativity on the positive half line holds by using just the interval $\left[0, T_{0}\right]$. As a starting guess for the Convex Optimisation algorithm an initial $\hat{\mathbf{b}}_{0} \in \hat{\mathcal{B}}(\hat{\mathbf{A}}, \hat{\mathbf{c}})$ is always available since the zero vector itself satisfies the non-negativity constraint. Using the BudanFourier algorithm it is possible to choose $\alpha, 0 \leq \alpha \leq 1$, such that $\hat{\mathbf{b}}_{0}=\alpha \hat{\mathbf{b}}^{*}$ lies on the boundary of $\mathcal{B}(\hat{\mathbf{A}}, \hat{\mathbf{c}})$.

Using a convex search algorithm for $i>0 \hat{\mathbf{b}}_{i}$ is updated to $\hat{\mathbf{b}}_{i+1}$ decreasing the criterion

$$
\left\|\hat{\mathbf{c}} e^{\hat{\mathbf{A}} x}\left(\hat{\mathbf{b}}_{i+1}-\hat{\mathbf{b}}^{*}\right)\right\|_{2}^{2}
$$

This step is repeated until no further improvement is possible implying the global minimum has been located at $\hat{\mathbf{b}}_{C}^{*}$. The essential result is that at each point, obtained in the algorithm, that is not equal to the global minimizing point, a direction exists in which the criterion function is improved. To see this consider the piece of straight line connecting a point obtained in the algorithm with the globally minimizing point. Due to convexity, the piece of straight line will lie wholly in the set $\hat{\mathcal{B}}(\hat{\mathbf{A}}, \hat{\mathbf{c}})$, while the criterion will be strictly decreasing along this straight line (until the global minimum is be reached). The convex optimization algorithm used tries to find a direction in which the criterion function is improved. The result states that such a direction can always be found except if we would have arrived at the global optimum. The algorithm is illustrated in Figure 3.

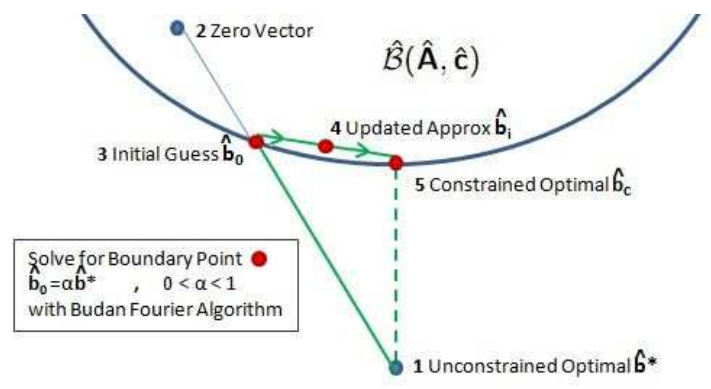

Fig. 3. Convex Optimisation Algorithm for Non-Negative EPT Density Function using Budan Fourier Technique

\section{Finite Interval to Test for Non-Negativity in presence of Strictly Dominant Pole}

Given an EPT function with the following minimal realization with strictly dominant real pole $\lambda_{M}<0$ and coefficient $\mu_{M}>$ 0 ,

$$
\left(\begin{array}{cc|c}
\lambda_{M} & 0 & 1 \\
0 & \hat{\mathbf{A}} & \hat{\mathbf{b}} \\
\hline \mu_{M} & \hat{\mathbf{c}} & 0
\end{array}\right)
$$

If $\hat{\mathbf{b}}=\hat{\mathbf{b}}^{*}$ then the triple $\left(\hat{\mathbf{A}}, \hat{\mathbf{b}}^{*}, \hat{\mathbf{c}}\right)$ minimizes the criterion in Eq. (17) for a given pair $(\hat{\mathbf{A}}, \hat{\mathbf{c}})$.

Define the non-empty set $\mathcal{B}_{1}(\hat{\mathbf{A}}, \hat{\mathbf{c}})$ for a pair $(\hat{\mathbf{A}}, \hat{\mathbf{c}})$ as follows: 


$$
\mathcal{B}_{1}(\hat{\mathbf{A}}, \hat{\mathbf{c}})=\left\{\hat{\mathbf{b}} \mid\left\|\hat{\mathbf{c}} e^{\hat{\mathbf{A}} x} \hat{\mathbf{b}}\right\|_{2} \leq 2\left\|\hat{\mathbf{c}} e^{\hat{\mathbf{A}} x} \hat{\mathbf{b}}^{*}\right\|_{2}\right\}
$$

Note that the minimising point $\hat{\mathbf{b}}_{C}^{*}$ will necessarily lie in the bounded set $\mathcal{B}_{1}(\hat{\mathbf{A}}, \hat{\mathbf{c}})$.

Proof

The zero vector lies in the convex set $\mathcal{B}(\hat{\mathbf{A}}, \hat{\mathbf{c}})$ and we also have $\hat{\mathbf{b}}_{C}^{*} \in \mathcal{B}(\hat{\mathbf{A}}, \hat{\mathbf{c}})$.

We let $q(x)=\hat{\mathbf{c}} e^{\hat{\mathbf{A}} x} \hat{\mathbf{b}}^{*}$ and $p(x)=\hat{\mathbf{c}} e^{\hat{\mathbf{A}} x} \hat{\mathbf{b}}_{C}^{*}$ and using the triangular inequality we can show that

$$
\|p(x)-q(x)\|_{2} \leq\|q(x)\|_{2} \Longrightarrow\|p(x)\|_{2} \leq 2\|q(x)\|_{2}
$$

\section{Proposition1}

For any minimal realization as described in Eq. (24) with strictly dominant real pole, $\lambda_{M}$ and $\hat{\mathbf{b}} \in \mathcal{B}_{1}(\hat{\mathbf{A}}, \hat{\mathbf{c}})$ we can construct a $T_{0}>0$ such that if $\hat{\mathbf{c}} e^{\hat{\mathbf{A}} x} \hat{\mathbf{b}} \geq-\mu_{M} e^{\lambda_{M} x}$ for all $x \in\left[0, T_{0}\right]$ then $\hat{\mathbf{c}} e^{\hat{\mathbf{A}} x} \hat{\mathbf{b}} \geq-\mu_{M} e^{\lambda_{M} x}$ for all $x \geq 0$.

\section{Proof}

The dominant pole constraint implies $\operatorname{Re}(\sigma(\hat{\mathbf{A}}))<\lambda_{M}$. Scaling the EPT density above by a factor $e^{-\lambda_{M} x}$ transforms the condition to be considered to

$$
\hat{\mathbf{c}} e^{\tilde{\mathbf{A}} x} \hat{\mathbf{b}} \geq-\mu_{M} \quad, \quad \forall x \geq 0
$$

where $\tilde{\mathbf{A}}=\left(\hat{\mathbf{A}}-\mathbf{I} \lambda_{M}\right)$ such that $\operatorname{Re}(\sigma(\tilde{\mathbf{A}}))<0$.

For any $\hat{\mathbf{b}} \in \mathcal{B}_{1}(\hat{\mathbf{A}}, \hat{\mathbf{c}})$ we have

$$
\left\|\hat{\mathbf{c}} e^{\tilde{\mathbf{A}} x} \hat{\mathbf{b}}\right\|_{2} \leq 2\left\|\hat{\mathbf{c}} e^{\tilde{\mathbf{A}} x} \hat{\mathbf{b}}^{*}\right\|_{2}
$$

$\mathbf{Q}$ is defined as the positive definite observability grammian

$$
\mathbf{Q}=\int_{0}^{\infty} e^{\tilde{\mathbf{A}}^{T} y} \hat{\mathbf{c}}^{T} \hat{\mathbf{c}} e^{\tilde{\mathbf{A}} y} d y
$$

The $L_{2}$ norm is then given by

$$
\hat{\mathbf{b}}^{T} \mathbf{Q} \hat{\mathbf{b}}=\left\|\hat{\mathbf{c}} e^{\tilde{\mathbf{A}} x} \hat{\mathbf{b}}\right\|_{2}^{2}
$$

By letting $\lambda_{\min }=\min \{\lambda \in \sigma(\mathbf{Q})\}$ such that

$$
\hat{\mathbf{b}}^{T} \mathbf{Q} \hat{\mathbf{b}} \geq \hat{\mathbf{b}}^{T} \hat{\mathbf{b}} \lambda_{\min }=\|\hat{\mathbf{b}}\|^{2} \lambda_{\min }
$$

it can be seen that

$$
\|\hat{\mathbf{b}}\|^{2} \lambda_{\min } \leq \hat{\mathbf{b}}^{T} \mathbf{Q} \hat{\mathbf{b}} \leq 4 D^{2}
$$

where $D^{2}=\hat{\mathbf{b}}^{* T} \mathbf{Q} \hat{\mathbf{b}}^{*}$. Hence there is an obvious bound on $\hat{\mathbf{b}}$

$$
\|\hat{\mathbf{b}}\|^{2} \leq \frac{4 D^{2}}{\lambda_{\min }}
$$

As the pair $(\tilde{\mathbf{A}}, \hat{\mathbf{c}})$ is observable and $\tilde{\mathbf{A}}$ an asymptotically stable matrix it must hold

$$
\lim _{x \rightarrow \infty} \hat{\mathbf{c}} e^{\tilde{\mathbf{A}} x} \rightarrow 0
$$

implying that $\forall \epsilon>0 \exists T_{0}>0$ s.t. $\forall x>$ $T_{0},\left\|\hat{\mathbf{c}} e^{\tilde{\mathbf{A}} x}\right\|^{2}<\epsilon$.

To find such a $T_{0}$ we define the Lyapunov function

$$
V(x)=\hat{\mathbf{c}} e^{\tilde{\mathbf{A}} x} \mathbf{M} e^{\tilde{\mathbf{A}}^{T} x} \hat{\mathbf{c}}^{T}
$$

where $\mathbf{M}$ is the positive definite solution to the Lypanunov equation

$$
\tilde{\mathbf{A}} \mathbf{M}+\mathbf{M} \tilde{\mathbf{A}}^{T}=-\mathbf{I}
$$

We see that $V(x)$ is monotonically decreasing

$$
V^{\prime}(x)=\hat{\mathbf{c}} e^{\tilde{\mathbf{A}} x}\left(\tilde{\mathbf{A}} \mathbf{M}+\mathbf{M} \tilde{\mathbf{A}}^{T}\right) e^{\tilde{\mathbf{A}}^{T} x} \hat{\mathbf{c}}^{T}<0
$$

$\forall x>0$. Letting $\tilde{\lambda}_{\text {min }}=\min \{\lambda \in \sigma(\mathbf{M})\}$ it is clear

$$
\begin{aligned}
\tilde{\lambda}_{\min }\left\|\hat{\mathbf{c}} e^{\tilde{\mathbf{A}} x}\right\|^{2} & \leq \hat{\mathbf{c}} e^{\tilde{\mathbf{A}} x} \mathbf{M} e^{\tilde{\mathbf{A}}^{T} x} \hat{\mathbf{c}}^{T} \\
\left\|\hat{\mathbf{c}} e^{\tilde{\mathbf{A}} x}\right\|^{2} & \leq \frac{V(x)}{\tilde{\lambda}_{\min }}
\end{aligned}
$$

We let

$$
K=\frac{4 D^{2}}{\tilde{\lambda}_{\min } \lambda_{\min }}
$$

For all $\hat{\mathbf{b}} \in \mathcal{B}_{1}(\hat{\mathbf{A}}, \hat{\mathbf{c}})$ we must have that

$$
K V(x) \geq \hat{\mathbf{c}} e^{\tilde{\mathbf{A}} x} \hat{\mathbf{b}} \hat{\mathbf{b}}^{T} e^{\tilde{\mathbf{A}}^{T} x} \hat{\mathbf{c}}^{T}
$$

We can then solve for $T_{0}$ such that

$$
V\left(T_{0}\right)=\frac{\mu_{M}^{2}}{K}=\frac{\mu_{M}^{2} \tilde{\lambda}_{\min } \lambda_{\min }}{4 D^{2}}
$$

proving Proposition 1.

\section{Non-Negative Convex Optimisation Algorithm Example}

We now provide an example to illustrate the performance of the non-negative convex optimisation algorithm described in Sections 5, 6 and 7. The function we will approximate is

$$
h(x)=16 e^{-\frac{x}{2}}-30 e^{-x}+15 e^{-2 x}=\mu_{M} e^{\lambda_{M} x}+\hat{\mathbf{c}} e^{\hat{\mathbf{A}} x} \hat{\mathbf{b}}^{*}
$$

The dominant pole $\lambda_{M}=-\frac{1}{2}$ and its coefficient $\mu_{M}=16$ are easily identifiable. The minimal realization of $h(x)$ in state space form is given by

$$
\left(\begin{array}{ccc|c}
-0.5 & 0 & 0 & 1 \\
0 & -1 & 0 & 1 \\
0 & 0 & -2 & 1 \\
\hline 16 & -30 & 15 & 0
\end{array}\right)=\left(\begin{array}{cc|c}
\lambda_{M} & 0 & 1 \\
0 & \hat{\mathbf{A}} & \hat{\mathbf{b}}^{*} \\
\hline \mu_{M} & \hat{\mathbf{c}} & 0
\end{array}\right)
$$

It is clear from Figure 4 that $h(x)$ is negative on the interval $[0.1897,0.6575]$. We will approximate $h(x)$ with the nonnegative EPT function $f(x)$

$$
f(x)=\mu_{M} e^{\lambda_{M} x}+\hat{\mathbf{c}} e^{\hat{\mathbf{A}} x} \hat{\mathbf{b}}_{C}^{*}
$$

For the pair $(\hat{\mathbf{A}}, \hat{\mathbf{c}})$ we will use the algorithm described in Section 5 to derive $\hat{\mathbf{b}}_{C}^{*}$ minimising the $L_{2}$ norm

$$
\min _{\{\hat{\mathbf{b}} \in \mathcal{B}(\hat{\mathbf{A}}, \hat{\mathbf{c}})\}}\left\|\hat{\mathbf{c}} e^{\hat{\mathbf{A}} x}\left(\hat{\mathbf{b}}^{*}-\hat{\mathbf{b}}\right)\right\|_{2}^{2}
$$

where the convex set $\mathcal{B}(\hat{\mathbf{A}}, \hat{\mathbf{c}})$ is as defined in Section 4 .

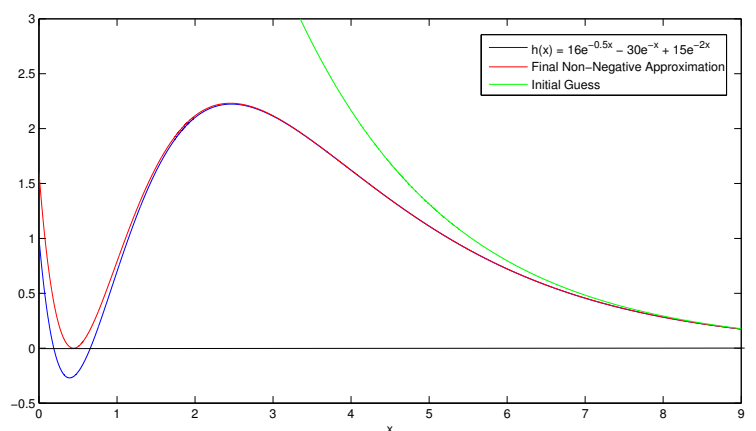

Fig. 4. Approximation of $h(x)$ (blue) with final non-negative function $f(x)$ (red) and the initial approximation (green)

Using the technique from Section 7 we can construct the finite interval on which it is necessary to check for non-negativity. In 
this instance the interval is $[0,9.2156]$. Hence if $f(x)$ is nonnegative on $[0,9.2156]$ then we know that $f(x)$ is non-negative on $[0, \infty)$.

We begin the convex optimisation with the initial guess, $\hat{\mathbf{b}}_{0}=$ $(0.9797,0.9797)^{T}$ which is a convex combination of the zero vector and $\hat{\mathbf{b}}^{*}$. Running the algorithm we find the $\hat{\mathbf{b}}_{C}^{*}$ minimising Eq. (45) is $\hat{\mathbf{b}}_{C}^{*}=(0.9984,1.0366)^{T}$ which yields an $L_{2}$ norm of 0.1296 . The non-negative approximating EPT function $f$ has a state space realization

$$
\left(\begin{array}{ccc|c}
-0.5 & 0 & 0 & 1 \\
0 & -1 & 0 & 0.9982 \\
0 & 0 & -2 & 1.0359 \\
\hline 16 & -30 & 15 & 0
\end{array}\right)=\left(\begin{array}{cc|c}
\lambda_{M} & 0 & 1 \\
0 & \hat{\mathbf{A}} & \hat{\mathbf{b}}_{C}^{*} \\
\hline \mu_{M} & \hat{\mathbf{c}} & 0
\end{array}\right)
$$

We can calculate the minimum of $f(x)$ which is a small positive number of the order $10^{-15}$ indicating that $\hat{\mathbf{b}}_{C}^{*}$ does indeed lie on the boundary of the $\operatorname{set} \mathcal{B}(\hat{\mathbf{A}}, \hat{\mathbf{c}})$. The optimisation procedure took 65 seconds to compute.

The convex optimisation and Budan-Fourier algorithms are available to download for Matlab from "www.2-ept.com".

\section{Concluding Remarks}

If we have $\hat{\mathbf{b}}^{*} \notin \mathcal{B}(\hat{\mathbf{A}}, \hat{\mathbf{c}})$ then we can find $\hat{\mathbf{b}}_{C}^{*} \in \mathcal{B}(\hat{\mathbf{A}}, \hat{\mathbf{c}})$ for a given pair $(\hat{\mathbf{A}}, \hat{\mathbf{c}})$ but it is still possible to adapt the $(\hat{\mathbf{A}}, \hat{\mathbf{c}})$ and derive a new $\hat{\mathbf{b}}_{C}^{*} \in \mathcal{B}(\hat{\mathbf{A}}, \hat{\mathbf{c}})$ to try and improve the non-negative approximation. Also further iterations can be used to determine the strictly dominant pole and the triple $\left(\hat{\mathbf{A}}, \hat{\mathbf{b}}_{C}^{*}, \hat{\mathbf{c}}\right)$ jointly. (We can use $h-\hat{\mathbf{c}} e^{\hat{\mathbf{A}} x} \hat{\mathbf{b}}_{C}^{*}$ to perform a log-linear regression or an $L^{2}$ optimal order one EPT approximation!) And finally the resulting values could be used as a starting point in a likelihood optimization procedure.

\section{Acknowledgements}

The authors would like to thank Fabien Seyfert and Finbarr Holland for their valuable contributions.

\section{References}

Anderson, B., Deistler, M., Farino, L., Benvenuti, L., NonNegative Realization of a Linear System With Non-Negative Impulse Response IEEE Transactions on Circuits and Sytems, Fundamental Theory and Applications, Vol 43, No. 2, February 1996

Hanzon, B., Sexton, C., State Space Calculations for twosided EPT Densities with Financial Modelling Applications. Submitted. Draft version available at "www.2-ept.com"

Hanzon, B., Holland, F. Non-Negativity of Exponential Polynomial Trigonometric Functions - A Budan Fourier Sequence Approach. BFS Toronto Poster 429, June 22 - 26, 2010. Online version available at "www.edgeworth.biz"

Hanzon, B., Holland, F. Non-negativity analysis for Exponential-Polynomial-Trigonometric Functions on the non-negative real half-line Forthcoming. Draft version available online at "www.edgeworth.biz", 2011

Olivi, M. Parametrization of Rational Lossless Matrices with Applications to Linear System Theory HDR Thesis, 2010

Hanzon, B., Olivi, M., Peeters, R.L.M, Balanced Realizations of Discrete-Time Stable All-Pass Systems and the Tangential Schur Algorithm Linear Algebra and its Applications, 2006
Marmorat, J.P., Olivi, M., Nudelman interpolation, parametrizations of lossless functions and balanced realizations. Automatica, 43:1329-1338, 2007.

Marmorat, J.P., Olivi, M., Seyfert, Identification of Microwave Filters by Analytic and Rational $H^{2}$ Approximation Forthcoming

Partington, J.R., Interpolation, Identification and Sampling Oxford University Press, 1997

\section{A Appendix: Derivation of Fourier Coefficients}

We begin by defining the Hardy space of the disk $L^{2}(\mathbb{T})$ which can be split into two orthogonal subspaces

$$
L^{2}(\mathbb{T})=H^{2} \oplus H_{\perp}^{2}
$$

where $H_{\perp}^{2}$ is the subspace of the Hardy space of the exterior of the disk consisting of strictly proper functions whose Fourier coefficients of non-negative index are zero.

We can represent the discrete time rational transfer function $\tilde{H}(z)$ defined on $H_{\perp}^{2}$ in terms of its Fourier coefficients as

$$
\begin{aligned}
\tilde{H}(z) & =\sum_{n=-\infty}^{0} c_{n} z^{n} \\
\tilde{H}\left(e^{i t}\right) & =\sum_{n=-\infty}^{0} c_{n} e^{i n t}
\end{aligned}
$$

where $c_{n}$ are the Fourier coefficients given by

$$
c_{n}=\frac{1}{2 \pi} \int_{0}^{2 \pi} \tilde{H}\left(e^{i t}\right) e^{-i n t} d t=<\tilde{H}, e^{i n t}>_{L^{2}(\mathbb{T})}
$$

The problem can be transformed into continuous time using the isometry from Eq. (6) and the Möbius transforms from Eq. (7)

$$
\begin{aligned}
H(s) & =\frac{\sqrt{2}}{s-1} \tilde{H}\left(\frac{s+1}{s-1}\right) \\
& =\frac{\sqrt{2}}{s-1} \sum_{n=-\infty}^{\infty} c_{n}\left(\frac{s+1}{s-1}\right)^{n}
\end{aligned}
$$

This is an isometry from $L^{2}(\mathbb{T})$ to $L^{2}(i \mathbb{R})$ as it sends the Hardy space $H_{\perp}^{2}$ onto $H^{2}\left(\mathbb{C}^{+}\right)$where $\mathbb{C}^{+}$denotes the open right half plane.

The Fourier coefficients are transformed as

$$
c_{n}=\frac{1}{2 \pi}<H, G_{n}>_{H^{2}\left(\mathbb{C}^{+}\right)}
$$

where

$$
G_{n}(s)=\sqrt{2} \frac{(s+1)^{n}}{(s-1)^{n+1}}
$$

Using Plancherel's Theorem we can transform the Fourier coefficients from $H^{2}\left(\mathbb{C}^{+}\right)$to $L^{2}(0, \infty)$ such that

$$
c_{n}=<h, g_{n}>_{L^{2}(0, \infty)}
$$

As we only consider the Fourier coefficients with $n<0$ we see that $g_{n}$ is the Fourier inverse of $G_{n}$ given by

$$
g_{n}(t)=\sqrt{2} e^{-t}\left(\sum_{j=0}^{-n-1}\left(\begin{array}{c}
-n-1 \\
j
\end{array}\right) \frac{(-2 t)^{j}}{j !}\right)
$$

The reader is referred to Partington [1997], especially Corollary 1.4.2, for more information on such orthonormal sequences of functions, and Marmorat et al [2012] for a more in depth discussion on the transformation from discrete to continuous time in a similar setting. 